\title{
On influence of substrate compliance on delamination and buckling of coatings
}

\author{
Konstantin B. Ustinov \\ A. Ishlinsky Institute for Problems in Mechanics RAS, Moscow, Russia \\ ustinov@ipmnet.ru
}

Keywords: coating, delamination, buckling, critical stress, plate, elastic clamping, elastic foundation.

\begin{abstract}
Problems related to delamination of coatings from substrates and their buckling are widely investigated due to importance of the problem for application in industry, particularly in micro- and nano-electronics and biology. The problem was addressed by many authors by using both analytical and numerical approaches.

Here the model is developed that treats the delaminated part of the coating as a plate with a special attention given to the boundary conditions, which are supposed in the form of the generalized elastic clamping, i.e. it is supposed that components of displacements and angle of rotation at the point of clamping are related to the acting total force and total moment by means of $3 \times 3$ matrix of elastic coefficients. Knowing the coefficients of this matrix allows determining parameters of buckling such as the critical stress, energy release rate due to further delamination, amplitude of buckling.

A few variants of analytical models to determine the coefficients of this $3 \times 3$ matrix were considered and discussed. Both the cases of isotropic and anisotropic coating and substrate are considered. The solution for some matrix coefficients was obtained with the help of Fourier transform and WienerHopf technique.

On the base of the obtained solution it is shown that the difference between the obtained critical stress and the critical stress of a rigidly clamped plate can be defined by a single nondimensional parameter, which is a combination of the elastic constants of the coating and substrate, and the ratio of delamination length to coating thickness. The results correlate well with the results obtained using the FEM model.
\end{abstract}

\section{Introduction}

The interest to effects, related to coatings delaminations causes by importance of the problem for such fields as micro- and nanoelectronics, biology, medicine. Number of studies is devoted to the problem e.g. [1]-[8]. In [2], [3], in particular, the problem of stability loss of coating delaminated from rectilinear rigid substrate was solved. The critical stress, magnitude of deflection [2], [3], and energy release rates along and across the delamination front as well were found. The delaminated part of coating was considered as a clamped plate, which corresponds to rigid substrates. However, detailed studies yield that, from the one hand, the condition of rigid clamping are not satisfied exactly even for absolutely rigid substrates [5], and, from the other hand, on the base of numerical calculations it is said [6], [7] that even for substrates tree times softer then coating the condition of rigid clamping yields acceptable errors. The last statement has to be accepted with cautions, because the influence of delamination size, importance of which being confirmed by other studies [5], [8], was not investigated in [6].

Influence of the substrate rigidity was studied by [4-6], however without paying attention to the influence of the delamination width to the critical stress. The analysis of influence of both substrate compliance and delamination width on the critical stress was performed in [5]. The delamination 
was modeled by a plate, with boundary condition corresponding to generalized elastic clamping, i.e. magnitudes of tangential displacements and gradient of deflection of the clamped edges were supposed to be proportional to tangential force and bending moment acting at the points of clamping. The coefficients of elastic clamping depending on two mentioned parameters were calculated by solving numerically a system of integral equations [9].

Contrary to [5], in [9] - [11] and here attempts were made to estimate the coefficients of elastic clamping analytically, and semi-analytically.

\section{Problem formulation}

Consider elastic half-plane (substrate) with adjusted layer (coating) of thickness $h$, which elastic properties are different from the properties of substrate. The layer is perfectly glued to the half-plane everywhere except a section of length, $2 b$, along which a delamination takes place. The Cartesian coordinate system is chosen so that $x$-axis be parallel the half-plane boundary, and $y$-axis coincide with its external normal, the origin of the coordinate frame coinciding with the delamination centre. Thus the half-plane occupies area $y<-h / 2$, the layer does $-h / 2<y<h / 2$, the delamination does $-b<y<b, y=-h / 2$. The Young moduli and Poisson ratios of the coating and substrate are $E, \nu, E_{s}, v_{s}$, respectively. The layer is assumed to be subjected to the tensile eigenstrain causing compressive stresses, $\sigma_{0}$, acting along the boundary. Such a situation takes place while heating the system in question if thermal extension of the layer is higher than the one of the half-plane. On reaching by the compressive stress some particular level of $\sigma^{\text {cr }}$, the loosing of stability occurs and the layer bends (Figure 1). The problem is to find the value of stress, $\sigma_{0}$, corresponding to the loss of stability.

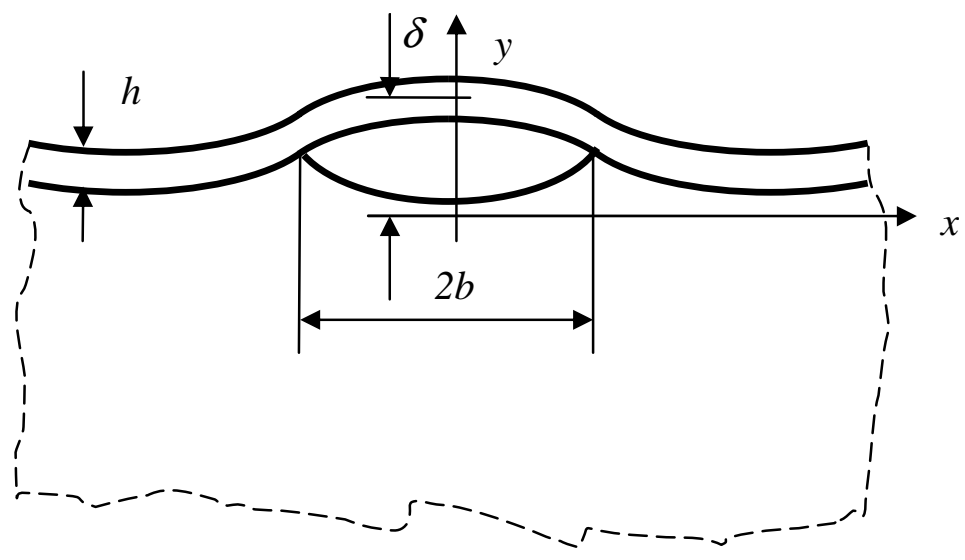

Fig. 1. Geometrical configuration.

The problem was solved in [6] numerically, using FEM, the value of $\sigma^{c r}$ being presented as products of corresponding values, calculated for the clamped plane $\sigma_{0}^{c r}$ and a coefficient $\gamma_{\sigma}$

$\sigma^{c r}=\sigma_{0}^{c r} \gamma_{\sigma}$ 
Magnitude of $\sigma_{0}^{c r}$ may be calculated using elementary methods. Thus for the clamped plate of length $2 b_{0}$ the critical stress is [12]:

$$
\sigma_{0}^{c r}=\frac{\pi^{2} h^{2} E^{*}}{12 b_{0}^{2}}, \quad E^{*}=\frac{E}{1-v^{2}}
$$

In [9], [10] and here estimations for coefficient $\gamma_{\sigma}$ are obtained on the base of the theory of plates.

\section{Model of simple elastic clamping}

In the framework of von Karman theory the plate deflection is

$$
E^{*} \frac{h^{3}}{12} v^{I V}(x)+\sigma(x) h v^{\prime \prime}(x)=0
$$

Here $\sigma(x)$ is the compressive stress within the cross-section of the coating. It should be noted that this stress is generally differ from $\sigma_{0}$ due to relaxation caused by curving of the delaminated part of the coating. For $-b<x<b, \sigma(x)=\sigma^{c r}=$ const the general solution of Eq. (3) is

$$
\begin{gathered}
v(x)=A_{1} \cos k x+A_{2},|x|<b \\
k=\sqrt{\frac{12 \sigma^{c r}}{E^{*} h^{2}}}
\end{gathered}
$$

Here constants $A_{i}$ have to be determined from the boundary conditions. As a variant of the solution it may be suggested that the delaminated section of coating could be treated as a plate with elastically clamped ends. Condition of elastic clamping at points $x= \pm b$ is

$$
\left.v^{\prime}(x)\right|_{x=b}=\left.h d v^{\prime \prime}(x)\right|_{x=b}
$$

Here $h d$ is the coefficient of proportionality between the angle of the plate at clamping and the second derivative of the displacement (proportional, in turn, to the bending moment, acting at this point); $d$ is a dimensionless coefficient that cannot be determined in the frame of elementary theory. The presence of plate thickness $h$ is due to necessity of adjusting dimensions: this parameter is the only one of the dimension of length in the model, because the clamping stiffness may not depend on the plate size $b$ (for more information see [9]-[11]). Substitution of Eq. (4) into Eq. (6) yield

$$
\tan k b+k h d=0
$$

The expression for the coefficient $d$ were obtained in [10] on the base of the model where the coating was considered as a plate and the substrate as half-plane. The solution was found with the 
help of Fourier transform and Wiener-Hopf technique. By neglecting the action of shear stress it was found

$$
d=d_{0} \sqrt[3]{E^{*} / E_{s}^{*}}, d_{0}=2^{2 / 3} 3^{-5 / 6} \approx 0,636
$$

Here

$$
E_{s}^{*}=E_{s} /\left(1-v_{s}^{2}\right), \quad E_{s}^{*}=2\left\{\beta_{22}\left[\beta_{66}+2\left(\beta_{12}+\sqrt{\beta_{11} \beta_{22}}\right)\right]\right\}^{-1 / 2}
$$

for isotropic and anisotropic (orthotropic) substrate, respectively; $\beta_{i j}$ are components of matrix of compliancy. For anisotropic (orthotropic) coating modulus $E^{*}$ is an independent material property. For rigid clamping

$$
k_{0}=\frac{\pi}{b}
$$

The substitution of Eqs. (1), (5), (8), (10) into Eq. (7) gives

$$
\tan \pi \sqrt{\gamma_{\sigma}}+d_{0} \beta \pi \sqrt{\gamma_{\sigma}}=0
$$

Here

$$
\beta=(h / b) \sqrt[3]{E^{*} / E_{s}^{*}}
$$

Dependence of $\gamma_{\sigma}$ on $\beta$ according to this model is presented on Fig. 2.

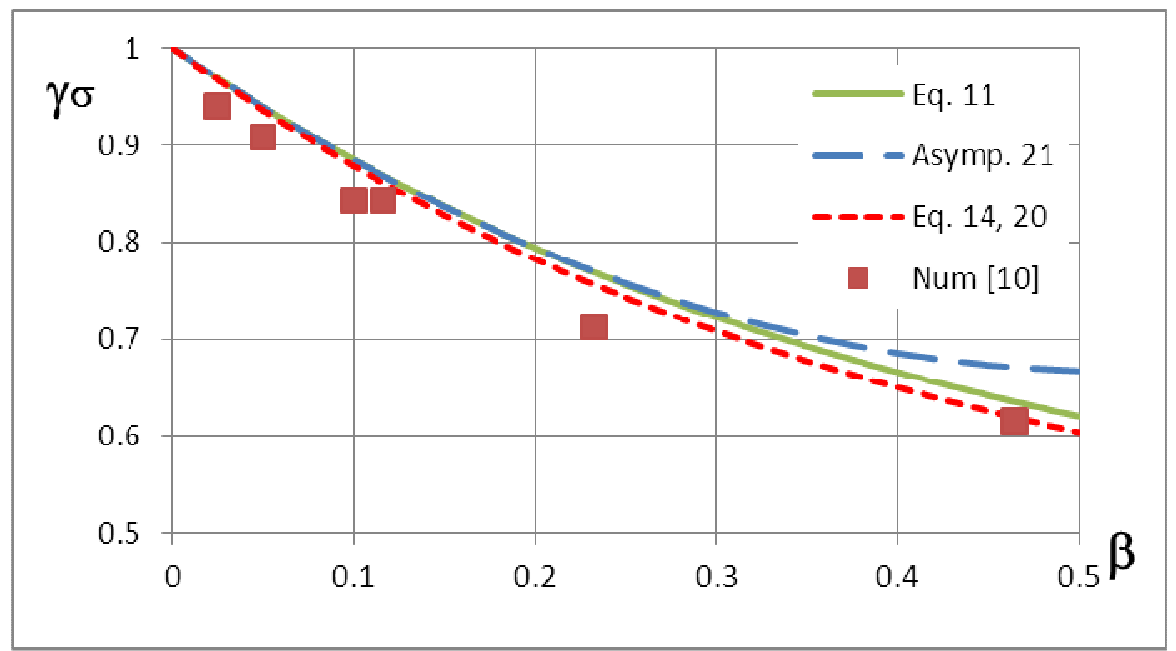

Fig. 2. Dependence of $\gamma_{\sigma}$ on $\beta$. 


\section{Model of generalized elastic clamping}

The model of elastic clamping may be generalized in order to account the influence of longitudinal force on the boundary conditions. Such a model was used by [5] for finding the critical stress of buckling of the delaminated coating. In the frame of that model it is supposed that longitudinal displacement $u$ and angle of rotation are linear function of longitudinal force $F$ and bending moment $M$ acting at the point of clamping

$$
\begin{aligned}
& E^{*} u=a_{11} F+a_{12} h^{-1} M \\
& E^{*} v^{\prime}=a_{12} h^{-1} F+a_{22} h^{-2} M
\end{aligned}
$$

Although condition of elastic clamping Eq. (6) was proven to be asymptotically correct [13], and other terms might be beyond the accuracy of beam theory, accounting for additional terms in Eq. (13) may be useful in numerical calculations.

The direct application of boundary condition Eq. (13) to Eq. (4) is impossible due to presence of unknown parameter $F$, which, however, may be found by solving the equation for longitudinal displacement $u$, simultaneously with Eq. (3). That was done in [5] with the final result for the critical stress after writing it in the form convenient for our purposes being:

$$
\frac{\tan \pi \sqrt{\gamma_{\sigma}}}{\pi \sqrt{\gamma_{\sigma}}}+\frac{h}{12 b}\left(a_{22}-\frac{a_{12}^{2}}{1+a_{11}}\right)=0
$$

The coefficient of symmetric matrix $a_{i j}$ of elastic clamping were calculated in [5] by numerical solving of a system of integral equations as functions of ratio of moduli of coating and substrate and ratio of coating thickness to the delamination length.

Taking into account that according to Eq. (12)

$$
a_{22}=12 d_{0} \sqrt[3]{E^{*} / E_{s}^{*}}
$$

expression Eq. (14) differ from Eq. (11) only by its last term, which is usually not large; nevertheless its accounting leads to (slight, but systematic) deviation of the results from the master curve, obtained by using more simple Eq. (11).

Dependence of $\gamma_{\sigma}$ on $\beta$ according to this model is also presented on Fig. 2, coefficient $a_{11}$ was calculated by with the help of the first formula of Eq. (20) for $E / E_{S}=1$.

If the initial surface is curved, then in addition to longitudinal force and bending moment, transverse force $N$ appears at the clamping point. Hence, condition Eq. (13) may be generalized:

$$
\begin{aligned}
& E^{*} u=a_{11} F+a_{12} h^{-1} M+a_{13} N \\
& E^{*} v^{\prime}=a_{12} h^{-1} F+a_{22} h^{-2} M+a_{23} h^{-1} N \\
& E^{*} v=a_{13} F+a_{23} h^{-1} M+a_{33} N
\end{aligned}
$$


Here matrix $a_{i j}$ may be called the extended matrix of coefficient of elastic clamping. Calculating coefficients of the extended matrix $a_{i j}$ is useful for studying delamination and buckling of initially curved coatings.

\section{Calculating coefficients of matrix of elastic clamping}

Coefficients of matrix (13) were calculated in [4] for a particular geometric parameters numerically, and in [5] by numerical solving a system of integral equations for various ratios geometric parameters and moduli. However it is desirable to have an asymptotical, or at least, approximating formulae.

Formulae for $a_{22}$ were obtained in [10] on the base of the model where the coating was considered as a plate and the substrate as half-plane by using Fourier transformation and Wiener-Hopf technique. The value is given by Eq. (8), Eq. (15). The value of $a_{23}$ were found (ibid.) to be

$$
a_{23}=2^{7 / 3} 3^{-2 / 3}\left(E / E_{s}\right)^{2 / 3} \approx 2.52\left(E / E_{s}\right)^{2 / 3}
$$

Coefficients $a_{11}, a_{12}$ (as well as some coefficients of the extended matrix Eq. (16)) for the case of coating and substrate being of the same material were obtained in [11]. The results were obtained on the base of solution of the matrix Wiener-Hopf problem [14], which was extended to describe displacements. The extraction of the coefficient $a_{22}$ for this case was made before [15], [16]. 3-D case was considered in [17].

It follows from the results of [5] that the value for $a_{12}$ does not effectively depend on the ratio elastic moduli, unless very soft substrates. For this case value

$$
a_{12}=\sqrt{3}
$$

was obtained [11]. It was also obtained (ibid.) the value for $a_{13}$ for the case of the same moduli of coating and substrate

$$
a_{13}=(1+\sqrt{3})
$$

The obtained formula for $a_{11}$ appeared rather awkward to be represented here.

An intent look at the results for $a_{11}$ reveals that all points with rather good accuracy may be represented as a single master curve (Fig 3). 


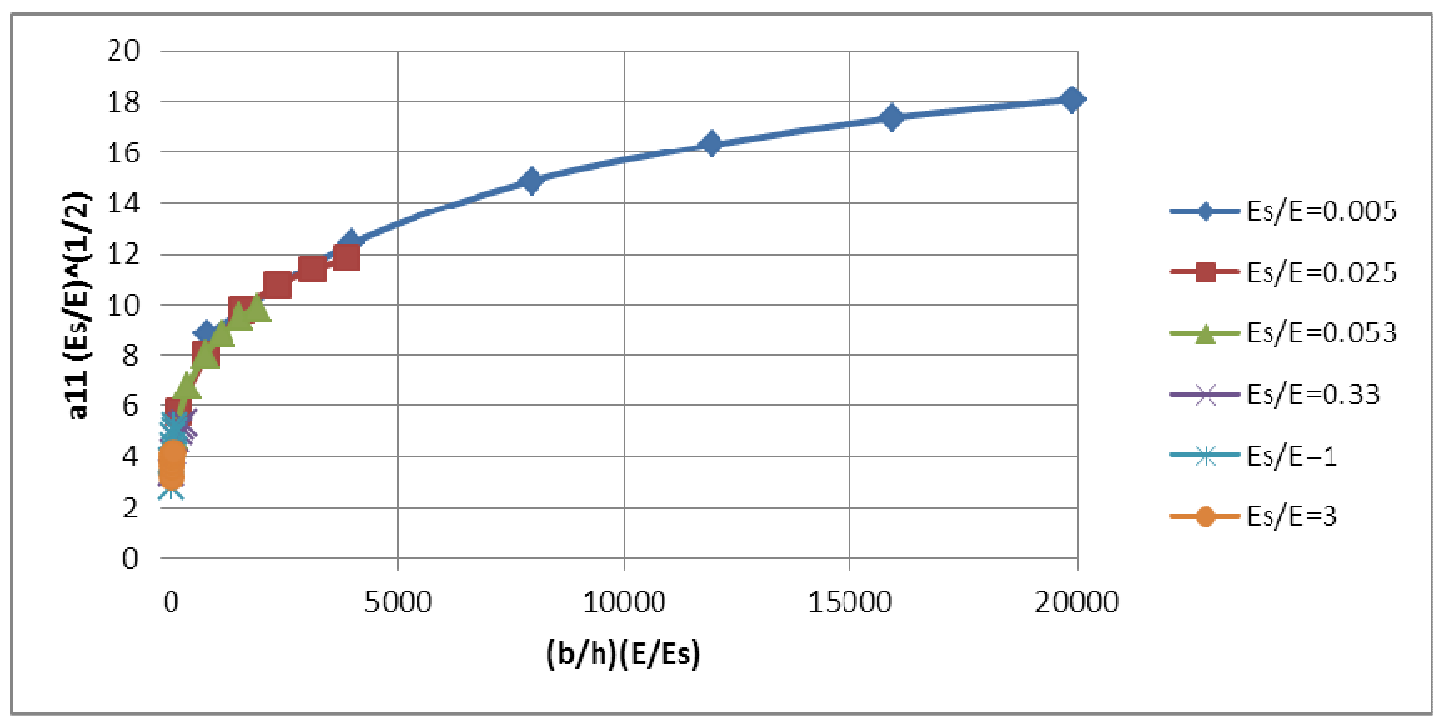

Fig. 3. Numerically obtained master curve for $a_{11}$ on the base of results [5].

The curve may be fitted by one of the following (purely approximating) formulae

$$
\begin{array}{ll}
a_{11}=\frac{1}{2} \sqrt{\frac{E}{E_{S}}+\frac{3}{2}\left[\frac{b}{h}\left(\frac{E}{E_{S}}\right)^{3}\right]^{1 / 4}} & a_{11}=\frac{3}{2}\left[\frac{b}{h}\left(\frac{E}{E_{S}}\right)^{3}\right]^{1 / 4} \\
a_{11}=3 \sqrt{\frac{E}{E_{S}}}\left[\left(\frac{b}{h} \frac{E}{E_{S}}\right)^{1 / 5}-1\right] & a_{11}=\sqrt{\frac{E}{E_{S}}}\left[4\left(\frac{b}{h} \frac{E}{E_{S}}\right)^{1 / 6}-3\right]
\end{array}
$$

\section{Some asymptotic estimations for critical stress}

For $\gamma_{\sigma}$ slightly diverging from unity, which corresponds to small $\beta$, asymptotic solution of Eq. (11) may be obtained by the following substitution

$$
\sqrt{\gamma_{\sigma}}=1+a_{1} \beta^{m}+a_{2} \beta^{n}+\ldots
$$

On expanding Eq. (21) into series for small $\beta$, the coefficients $a_{1}, a_{2}$, as well as exponents $m, n$ for which the solution exists are found. The corresponding solution is

$$
\sigma^{c r}=\frac{\pi^{2} h^{2} E^{*}}{12 b^{2}}\left(1-2 \beta+3 \beta^{2}+\right)
$$

Dependence of $\gamma_{\sigma}$ on $\beta$ according to this model is also presented on Fig. 2. 


\section{FEM calculations}

The carried FE analysis [9], the results of which are presented on Figure 2 (dots), confirms the analytically obtained dependence of the critical stress on the elastic and geometric parameters according to the suggested models.

\section{Summary}

The problem on loss of stability of a delaminated coating due to presence of intrinsic compressive deformations caused by, for example, heating is considered. The analytical solutions are obtained within the framework of classical thin plate theory. A simple expression for the critical compressive stress expression is found. It is shown that the ratio of critical stress to the critical stress for rigidly pinched plate is determined by a single nondimensional parameter, being a combination of elastic constants of the coating and substrate along with ratio of delamination length to coating thickness. The work was done under financial support of Program of Presidium of RAS N23 and RFBR (project 08-01-00860).

\section{References}

[1] J.W. Obreimoff: Proc. Roy. Soc., London, Vol. 127 A (1930) p. 290.

[2] L.M. Kachanov: Delamination Buckling of Composite Materials (Kluwer 1988)

[3] J.W.Hutchinson, Z. Suo, in Advances in Applied Mechanics edited by J. W. Hutchinson and T. Y. Wu, Vol. 29, (1992) p. 63.

[4] B. Cotterell, Z. Chen: Int. J. Fract. Vol. 104, (2000) p. 169.

[5] H.H. Yu, J.W. Hutchinson: Int. J. Fracture Vol. 113 (2002) p. 39.

[6] G. Parry, J. Colin, C. Coupeau, F. Foucher, A. Cimetière, J. Grilhé: Acta Mater. Vol. 53 (2005) p. 441.

[7] S. Faulhaber, C. Mercera, M.-W. Moon, J.W. Hutchinson, A.G. Evans: J. Mech. Phys. Solids, Vol. 54 (2006) p. 1004.

[8] H.H. Yu, M.Y. He, J.W. Hutchinson: Acta Mater. Vol. 49, (2001) p. 93.

[9] R.V. Goldstein, K.B.Ustinov, A.V.Chentsov: Computational mechanics. Vol. 4 ( 2011) N3. p. 48.

[10] R.L. Salganik, K.B. Ustinov: Izv RAS MTT N4 (2012) p. 50.

[11] K.B. Ustinov. Once more on a problem of a half plane, weakened by semi-infinite crack parallel to its boundary. Preprint IPM RAS (2012) in print

[12] S.P. Timoshenko: Strength of materials. Vol. 2. (Moscow, Nauka, 1965).

[13] K.B. Ustinov: Izv RAS MTT N3. (2008) p. 182.

[14] A.N. Zlanin, A.A. Khrapkov: DAN SSSR Vol. 31 (1986) N3 p. 1009.

[15] Ustinov, K.B., A.V. Dyskin, L.N. Germanovich: in 3rd Int. Conf. Localized Damage 94 (1994) p. 623.

[16] Dyskin, A.V., L.N. Germanovich, K.B. Ustinov: Int. J. Sol. Struc. Vol. 37 (2000) p.857.

[17] R.V. Golstein, A.B. Konovalov: Asymptotic analysis of 3-D problem on crack-delamination in two layered plate. Preprint IPMech RAS N 565 (1996). 